\title{
Rhabdomyosarcoma mimicking Acute Leukemia in a 42-year-old female
}

\author{
Rakul Nambiar K*, Anoop TM and Prakash NP \\ Department of Medical Oncology, Regional Cancer Centre, Trivandrum 695011, India
}

\begin{abstract}
Rhabdomyosarcoma (RMS) is a highly malignant soft tissue sarcoma that typically affects children and adolescents. Histologic appearance of RMS is similar to that of other small round blue cell tumors of childhood. Thus, RMS can mimic acute leukemia, both clinically and histologically. We report a case of a 42 -year-old female patient who presented with pancytopenia. She was referred to our centre with a diagnosis of acute leukemia after bone marrow studies. Bone marrow sample was reviewed at our centre, which showed atypical cells resembling blasts. However, myogenin positivity by immunohistohemistry in bone marrow specimen confirmed the diagnosis of RMS infiltrating bone marrow.
\end{abstract}

\section{Introduction}

Bone marrow infiltration by the non-hematopoietic malignancies poses a diagnostic dilemma because these patients usually present with clinical features attributable to cytopenias. Such patients usually are evaluated for a hematologic condition and thus there is usually delay in making the correct diagnosis. Neoplastic cells especially of small round cell neoplasms resemble the blasts of acute leukemia [1-3]. Cases of RMS presenting with disseminated disease without other obvious primary tumor present a diagnostic problem. Though RMS is a common soft tissue sarcoma in children younger than 15 years the incidence of RMS in adults is much lower after the age of 45 (average prevalence of 2-5\%).There are also reports of RMS confined to the bone marrow $[4,5]$. We present a case of RMS with bone marrow metastasis at the initial presentation, thus clinically and morphologically mimicking an acute hematological malignancy.

\section{Case report}

A 42-year-old female patient with dyspnea on exertion and purpurae was evaluated at a local hospital and was detected to have pancytopenia Peripheral smear examination revealed leukoerythroblastic blood picture. BM aspirates showed $76 \%$ of blast-like abnormal cells (Figure 1 ). She was referred to our centre with a diagnosis of acute leukemia. Laboratory investigations at our centre revealed hemoglobin- $6.8 \mathrm{~g} \%$, total count $-3800 / \mathrm{mm}^{3}$, platelet count $-8000 / \mathrm{mm}^{3}$. Morphologically, bone marrow aspirates revealed diffusely arranged vague clusters of large atypical cells with moderate amount of cytoplasm, some with cytoplasmic vacuolations. However, immunohistochemistry of bone marrow biopsy showed the positivity of tumor cells for myogenin (Figure 2). Thus, diagnosis of RMS infiltrating bone marrow was made. She was started on combination chemotherapy with vincristine, dactinomycin, and cyclophosphamide. Her symptoms decreased and her blood counts improved. The patient is currently doing well and she is undergoing chemotherapy.

\section{Discussion}

RMS is the most common soft tissue sarcoma of children and adolescents commonly involving head and neck region, urogenital tract and extremities. RMS may metastasize to the BM [6-8], but extensive marrow involvement is extremely rare [9]. Morphological and clinical resemblance of metastatic RMS to acute leukemia can result in misdiagnosis. We described a case of RMS masquerading acute leukemia, in which the diagnosis was made on the basis of immunochemistry. Bone marrow metastasis of RMS reveals a loosely arranged distribution of cells resembling blasts thus simulating acute leukemia. There are case reports of RMS misdiagnosed and treated as poorly differentiated leukemia based on morphology and cytochemistry alone and then proved as RMS based on demonstration of $t(2,13)$ by cytogenetics study [5].

Nonhematolymphoid tumors presenting in leukemic phase,

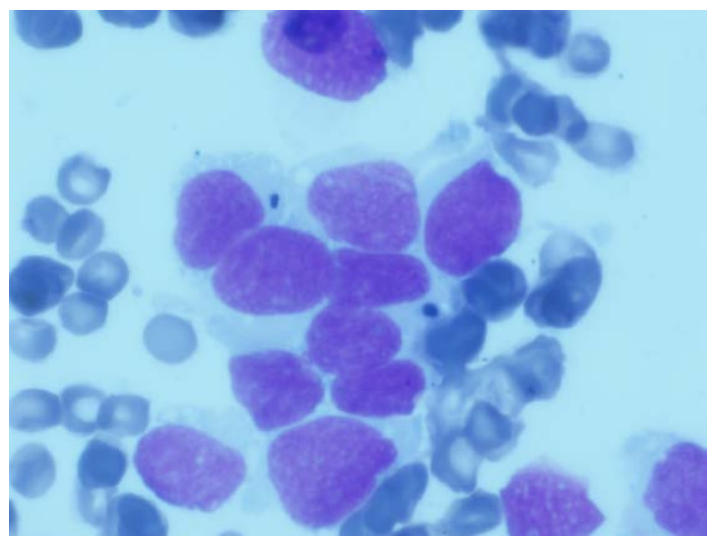

Figure 1. Bone marrow aspirate diffusely arranged and vague clusters of large atypical cells with moderate amount of cytoplasm, some with cytoplasmic vacuolations.

Correspondence to: Rakul Nambiar K, Senior Resident, Department of Medical Oncology, Regional Cancer Centre, Trivandrum 695011, India, E-mail: rakulnambiar@yahoomail.com

Key words: acute leukemia, small round blue cell, rhabdomyosarcoma

Received: May 04, 2017; Accepted: May 15, 2017; Published: May 17, 2017 


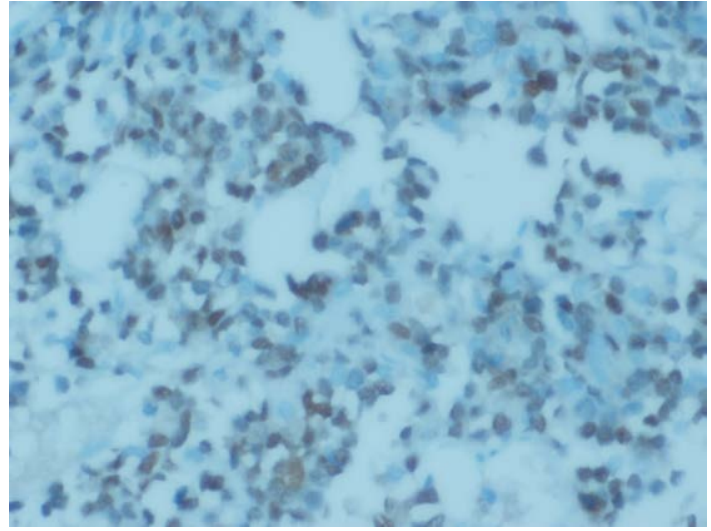

Figure 2. IHC showed abnormal cells positive for myogenin.

diffuse infiltration of bone marrow or inapparent primary site can be mistaken for hematolymphoid malignancies. Patients with widespread RMS can present with systemic symptoms, anemia, thrombocytopenia, disseminated intravascular coagulation, tumor lysis syndrome and thus clinically mimicking a hematologic malignancy. In cases of massive marrow involvement of solid tumors, such as the present cases, acute undifferentiated leukemia (AUL) should be considered. The blasts of AUL lack any morphologically differentiating features and they are usually negative for the lineage specific markers, such as cCD79a, cCD22, CD3, and/or CCD3 and MPO, while they are often positive for CD34, HLA-DR, CD38, CD7, and terminal deoxynucleotidyl transferase. In conclusion, RMS must be considered in the differential diagnosis of patients who present with clinical features of acute leukemia with atypical features in bone marrow studies.

\section{References}

1. Srinivas U, Pillai L, Kar R, Mahapatra M, Gujra S, et al. (2007) A case of rhabdomyosarcoma masquerading as acute leukemia at presentation: A case report. Indian J Pathol Microbiol 50: 917-919.

2. Shinkoda Y, Nagatoshi Y, Fukano R, Nishiyama K, Okamura J (2009) Rhabdomyosarcoma masquerading as acute leukemia. Pediatr Blood Cancer 52: 286287. [Crossref]

3. Naithani R, Kumar R, Mahapatra M, Agrawal N, Saxena R, et al. (2007) Pelvic alveolar rhabdomyosarcoma with bone marrow involvement misdiagnosed as acute myeloid leukemia. Hematol Oncol 24: 153-155.

4. Karagiannis P, Guth N, Thoennissen GB, Bern C, Sperveslage J, et al. (2015) Alveolar rhabdomyosarcoma confined to the bone marrow with no identifiable primary tumour using FDG-PET/CT. Clinical Sarcoma Research 5: 24.

5. Sandberg AA, Stone JF, Czarnecki L, Cohen JD (2001) Hematologic masquerade of rhabdomyosarcoma. Am J Hematol 68: 51-57.

6. Breneman JC, Lyden E, Pappo AS, Link MP, Anderson JR, et al. (2003) Prognostic factors and clinical outcomes in children and adolescents with metastatic rhabdomyosarcoma-A report from the intergroup rhabdomyosarcoma study IV. J Clin Oncol 21: 78-84. [Crossref]

7. Ruymann FB, Newton WA Jr, Ragab AH, Donaldson MH, Foulkes M (1984) Bone marrow metastases at diagnosis in children and adolescents with rhabdomyosarcoma. A report from the intergroup rhabdomyosarcoma study. Cancer 53: 368-373. [Crossref]

8. Carli M, Colombatti R, Oberlin O, et al. (2004) European intergroup studies (MMT489 and MMT4-91) on childhood metastatic rhabdomyosarcoma: Final results and analysis of prognostic factors. J Clin Oncol 22: 4735-4742. [Crossref]

9. Etcubanas E, Peiper S, Stass S, Green A (1989) Rhabdomyosarcoma, presenting as disseminated malignancy from an unknown primary site: a retrospective study of 10 pediatric cases. Med Pediatr Oncol 17: 39-44.

Copyright: (C2017 Rakul Nambiar K. This is an open-access article distributed under the terms of the Creative Commons Attribution License, which permits unrestricted use, distribution, and reproduction in any medium, provided the original author and source are credited. 